\title{
YBCO HIGH-TEMPERATURE SUPERCONDUCTING FILTERS ON M-PLANE SAPPHIRE SUBSTRATES
}

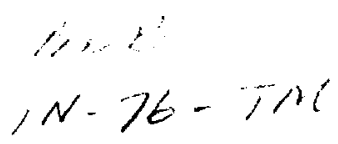

\author{
J. C. Sabataitis, ${ }^{*}$ C. H. Mueller," ${ }^{*}$ F. A. Miranda, J. Warner, \\ and $\mathrm{K}$. B. Bhasin \\ National Aeronautics and Space Administration \\ Lewis Research Center \\ Cleveland, Ohio, 44135, USA
}

\begin{abstract}
Since the discovery of High Temperature Superconductors (HTS) in 1986, microwave circuits have been demonstrated using HTS films on various substrates. These HTS-based circuits have proven to operate with less power loss than their metallic film counterparts at 77 $\mathrm{K}$. This translates into smaller and lighter microwave circuits for space communication systems such as multiplexer filter banks. High quality HTS films have conventionally been deposited on lanthanum aluminate $\left(\mathrm{LaAlO}_{3}\right)$ substrates. However, $\mathrm{LaAlO}_{3}$ has a relative dielectric constant $\left(\epsilon_{\mathrm{r}}\right)$ of 24 . With a $\epsilon_{\mathrm{r}}-9.4-11.6$, sapphire $\left(\mathrm{Al}_{2} \mathrm{O}_{3}\right)$ would be a preferable substrate for the fabrication of HTS-based components since the lower dielectric constant would permit wider microstrip lines to be used in filter design, since the lower dielectric constant would permit wider microstrip lines to be used for a given characteristic impedance $\left(Z_{41}\right)$, thus lowering the insertion losses and increasing the power handling capabilities of the devices. We report on the fabrication and characterization of $\mathrm{YBa}_{2} \mathrm{Cu}_{3} \mathrm{O}_{7-8}(\mathrm{YBCO})$ on $\mathrm{M}$ plane sapphire bandpass filters at $4.0 \mathrm{GHz}$. For a YBCO "hairpin" filter, a minimum insertion loss of $0.5 \mathrm{~dB}$ was measured at $77 \mathrm{~K}$ as compared with $1.4 \mathrm{~dB}$ for its gold counterpart. In an "edge-coupled" configuration, the insertion loss went down from $0.9 \mathrm{~dB}$ for the gold film to $0.8 \mathrm{~dB}$ for the YBCO film at the same temperature.
\end{abstract}

\section{INTRODUCTION}

Multiplexer filter banks used for channel separation in communication satellites require components that are as small and lightweight as possible. The filters used in multiplexers have

"Student, Electrical Engineering and Applied Physics Department, Case Western Reserve University, Cleveland, Ohio; work performed as NASA/OAI Summer Intern

"Now at Superconducting Core Technologies, Inc., Golden, Colorado

Advances in Cryogenic Engineering, Vol, 41 
been successfully made using waveguide or coaxial techniques, yet are large and heavy. Microstrip filters using conventional conductors, which potentially can be used in filter banks, are small, but are often too lossy for multiplexer applications, while conventional superconductors are less lossy, but have to be cooled with liquid helium to $4-5 \mathrm{~K}$. There would be many advantages in developing circuits that could operate at $77 \mathrm{~K}$, since these could be cooled at less cost with liquid nitrogen (boiling point of $77 \mathrm{~K}$ ) The discovery of High Temperature Superconducting (HTS) materials has brought a solution to these problems. Microstrip filters using HTS films are just as small and lightweight, but can display even less power loss than their waveguide or coaxial counterparts ${ }^{1-3}$. Considering that filter banks are composed of many filters, the gains achieved by using a lower-loss filter are often multiplied.

HTS thin films used in microstrip filters have been deposited on substrates such as Lanthanum Aluminate ( $\left.\mathrm{l}, \mathrm{AlO}_{3}\right)$, Magnesium Oxide ( $\left.\mathrm{MgO}\right)$, Neodymium Gallate $\left(\mathrm{NdGaO}_{3}\right)$, and sapphire $\left(\mathrm{Al}_{2} \mathrm{O}_{3}\right)$. In general, the best performance of HTS-based microwave components has been obtained from films deposited on $\mathrm{LaAlO}_{3}$. This material, however, has a relatively high dielectric constant, $\epsilon_{1} \sim 24^{+}$. Sapphire $\left(\mathrm{Al}_{2} \mathrm{O}_{3}\right)$ has a much lower dielectric constant of 9.411.6, depending on crystal orientations, and would be a better choice for a substrate. The lower dielectric constant would permit wider microstrip lines to be used for a given characteristic impedance $\left(Z_{0}\right)$, thus lowering the insertion losses and increasing the power handling capabilities of the devices. Although power handling capabilities of microstrip filters using high $\epsilon_{\mathrm{r}}$ substrates (such as $\mathrm{LaAlO}_{3}$ ) have been improved by using low $\mathrm{Z}_{0}$ resonance elements, the input power is limited by current crowding at junction between the feed line and first resonator. In addition, sapphire has a comparable loss tangent $\left(\operatorname{tam} \delta-4 \times 10^{-8}\right)$ to that of $\mathrm{LaAlO}_{3}\left(\tan \delta-0.76 \times 10^{-5}\right)$ at $77 \mathrm{~K}$ and $10 \mathrm{GHz}^{5}$. This combination of a lower dielectric constant and a low loss tangent results in an overall lower power loss 3

One slight drawback to the use of sapphire as a substrate, however, is the fact that YBCO film cannot be deposited successfully directly on the substrate due to the reactivity of the two materials. At the temperature required for deposition, sapphire reacts with barium oxide $(\mathrm{BaO})$ to form barium aluminate $\left(\mathrm{BaAl}_{2} \mathrm{O}_{4}\right)$. Therefore a buffer layer such as $\mathrm{SrTiO}_{3}$ or $\mathrm{MgO}$, which are both unreactive with the two materials, must be deposited on sapphire before the YBCO film can be deposited. Unfortunately, this creates some loss due to the resultant lattice mismatch. This loss, however, should be minimal enough that the gains from using an HTS film should more than compensate for the loss due to crystal lattice mismatch ${ }^{6}$.

HTS films have been successfully deposited on both (1TOO)- and (IIO2)- oriented sapphire (M- and R-plane, respectively). The optic axis in M-plane sapphire is aligned parallel to the substrate surface, whereas in R-plane sapphire it is oriented 32.4 degrees from the substrate surface. Circuit design is complicated by the dielectric anisotropy of the substrate, and becomes most challenging for substrate orientations for which the optic axis is neither perpendicular or parallel to the substrate plane (such as R-plane sapphire). Recent work ${ }^{7}$ has shown that the unloaded $Q$ values of linear, one-half wavelength resonators patterned on $M$ plane sapphire are considerably higher than on R-plane (2100 versus 600$)$. Since the low $Q$ values on the R-plane sapphire could not be attributed to conductor or dielectric losses, the only possibility is that the radiation losses in R-plane sapphire were higher than on M-plane sapphire. The difference in $Q$ values was attributed to radiation losses associated with the fact that the optic axis in R-plane sapphire is neither parallel nor perpendicular to the optic axis, while the optic axis for $M$-plane sapphire is in the plane of the substrate. Thus, $M$-plane sapphire is a better candidate for microwave circuits.

In the following sections, we will present design and test results of microwave 3-pole bandpass filters. Four filters were made using two basic designs. Each design was fabricated on M-Plane sapphire substrates using gold or YBCO films. (The YBCO films were obtained from a commercial vendor.) All four were tested at $77 \mathrm{~K}$, and display low-loss performance 


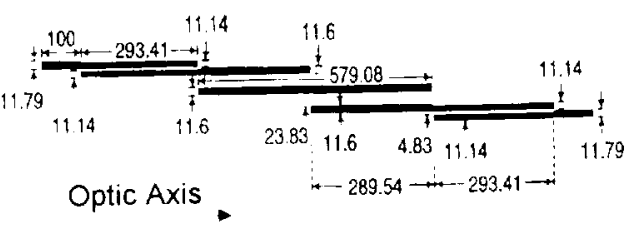

All dimensions in mils

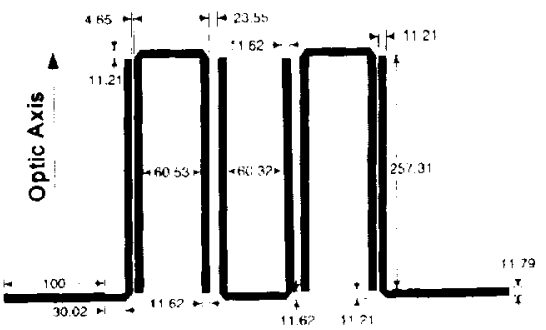

(Symmetrical About X-Axis)

(b) All dimensions in mils

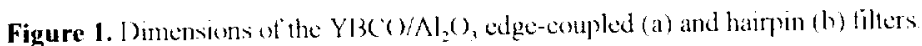

\section{FILTER DESIGN, FABRICATION, AND TESTING}

Two types of bandpass filters were designed using Eagleware software The filters were designed to resonate at $4.0 \mathrm{GHz}$ on M-plane sapphire with a dielectric constant of 9.4 . One was a hairpin shape and the other was an edge-coupled design (figure 1) Both were twoport, three-pole Chebyshev configurations with 50 -ohm lines and 4 percent bandwidths. Four filters were fabricated in a clean room from photo-reduced masks using standard photolithography and chemical etching techniques two using gold film and ground planes (both 2.5 microns thick), and two using single-sided YBCO film and gold ground planes. The gold filters were fabricated on 17-mil thick sapphire substrates. Since the YBCO films were deposited on 12 mil thick substrates, the linewidths and gaps were scaled to yield identical center frequencies and bandwidths. The sapphire used for the $\mathrm{YBCO}$ filter had a buffer layer of $\mathrm{MgO}$ and had a $3000 \AA$ thick YBCO film deposited by magnetron sputtering 8 , A $4000 \AA$ thick patch of gold 80 microns narrower than the width of the feedline was deposited on the edges of the YBCO feedlines to improve coupling from the coaxial pin to the filter. None of the YBCO filters were post-annealed, since YBCO on M-plane sapphire films have previously responded unfavorably to this process, becoming substantially more susceptible to thermallyinduced microcracks ${ }^{7}$. The edge-coupled filters were fabricated with feedlines parallel to the optic axis, and the hairpin filters were made with feedlines orthogonal to the optic axis, so that the coupling between resonant elements would be perpendicular to the optic axis, and hence the dielectric constant seen by the field lines in the coupling direction would be 9.4

The filters were tested using a Hewlett-Packard 8510C Automated Network Analyzer (ANA). Both were mounted on brass test fixtures with coaxial pin launchers. The test fixture was mounted inside the vacuum can of a closed-cycle helium gas refrigerator and fed a signal by an HP8517B S-parameter test set. The temperature was controlled using a Lake Shore DRC $91 \mathrm{C}$ temperature controller. Both the temperature and ANA were controlled by an HPBASIC program to record data. The ANA cables and connectors to the launchers on the test fixture were calibrated at room temperature $(298 \mathrm{~K})$ using a short-open-load-through calibration technique. An HP 9000-216 computer recorded measurements under vacuum (below 1 millitorr) and in the $20 \mathrm{~K}$ to $100 \mathrm{~K}$ temperature range. Data at $77 \mathrm{~K}$ was particularly used for insertion loss and unloaded $\mathrm{Q}$ analysis. Two s-parameters were recorded as a function of frequency, $S_{11}$ (reflection), and $S_{21}$ (transmission). The insertion losses at the maximum of the transmission parameters and at the midband points were recorded. The unloaded $Q$ was calculated using the following equation

$$
Q \cdot \frac{8.68\left(x_{3} \omega_{1}\right.}{m\left(\Delta I_{1}\right)_{11}}
$$




\begin{tabular}{lcc} 
& Insertion Loss (d13) & Unloaded $Q$ \\
\hline Gold flarpin Filter & 1.4 & 421 \\
YBCO Hairpin Filter & 0.5 & - \\
Gold lidge-coupled Filter & 0.9 & 368 \\
YBCO Fidge-coupled Filter & 0.8 & - \\
\hline
\end{tabular}

where $\omega_{1}{ }^{\prime}$ is the low pass prototype cutoff frequency (normally $=1$ ), w is the percent bandwidth, $\left(\Delta L_{2}\right)_{0}$ is the minimum insertion loss in $\mathrm{dB}$ (assuming a symmetrical and flat passband), and $C_{n}$ is the constant dependent on the Chebyschev ripple and filter order ${ }^{9}$. The constant 8.686 is a conversion from nepers to $\mathrm{dB}$

\section{RESULTS}

The insertion losses at $77 \mathrm{~K}$ for edge-coupled and hairpin filters are shown in Table I The insertion loss of the hairpin filter changed more drastically when the material was switched to YBCO than did the loss of the edge-coupled filters At $77 \mathrm{~K}$, the insertion loss of the gold hairpin filter was $1.4 \mathrm{~dB}$. When the YBCO hairpin filter was tested at $77 \mathrm{~K}$, the insertion loss went down to $0.5 \mathrm{~dB}$. The YBCO edge-coupled filter performed very well at lower temperatures. We observed an insertion loss of less than $0.3 \mathrm{~dB}$ at $35 \mathrm{~K}$. However, the loss went up to $0.8 \mathrm{~dB}$ at $77 \mathrm{~K}$, which was not significantly different from the gold edge coupled filter with a loss of $0.9 \mathrm{~dB}$ (also at $77 \mathrm{~K}$ )

The unloaded $Q$ values of the gold filters were around 400 (table 1) The unloaded $Q$ values were not calculated for the YBCO filters. These filters had very asymmetrical transmission parameters, unlike the gold versions (figures 2 and 3 ). All four filters had center frequencies around $4 \mathrm{GHz}$, but for the HTS filters, the point of minimum insertion loss was shifted a bit higher than the center frequency, making calculation of the unloaded $Q$ using equation ( 1 ) difficult. The assymetrical shape and poor performance of the filters were attributed to radiation losses associated with the substrate's anisotropy.

(dB)

Gold/Sapphire Hairpin Filter

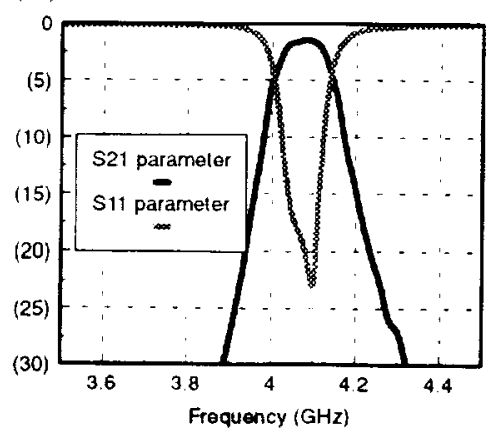

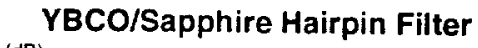

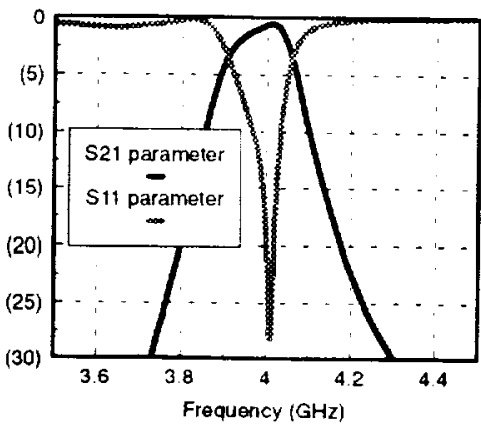

Figure 2. Reflection and transmission parameters of the gold and $\mathrm{YBCO}$ hairpin filters. 


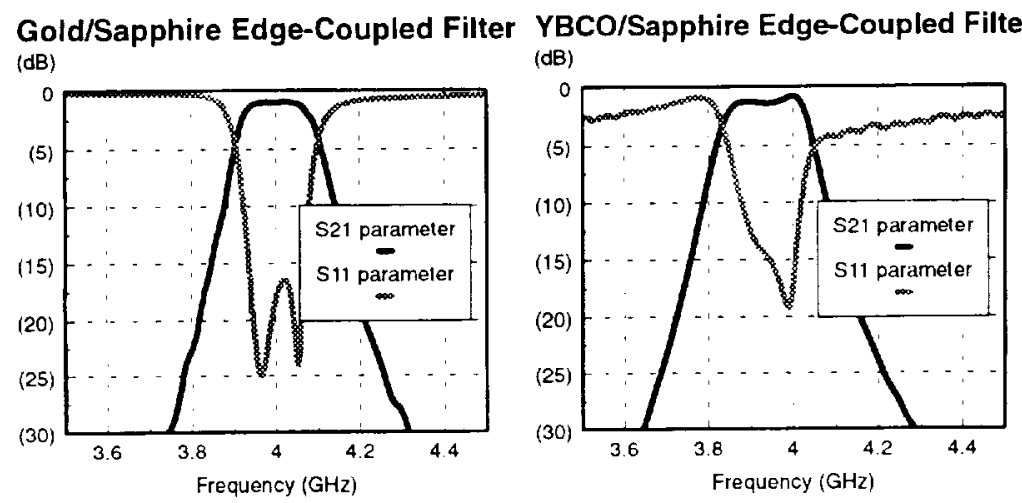

Figure 3. Reflection and transmission parameters of gold and YI3C ( ) edge-coupled filters

\section{CONCLUSIONS}

HTS YBCO filters have been fabricated on sapphire substrates because of its lower dielectric constant and loss tangent. We attribute the poor performance of the filters to radiation losses resulting from the dielectric anisotropy of the substrates. Despite our efforts to minimize the effects of dielectric anisotropy by aligning the resonator elements parallel to the optic axis, the performance for the YBCO filters is only slightly better than their gold counterparts. Because of this, only a small improvement was obtained by using low-loss YBCO films as the conductors. Since the $Q$ value of a single, one-half wavelength long resonator was measured to be $21000^{7}$, we conclude that most of the microwave energy in the three-pole filters was lost in coupling between elements.

\section{ACKNOWLEDGEMENT}

We would like to thank the NASA Lewis Technical Support Staff for their support in fabrication. In particular, we would like to thank Donna Bohman for deposition and etching of the films. We would also like to thank George Readus Jr. for making the test fixtures Finally, we are grateful to Linda D'Evelyn for helpful suggestions pertaining to the $Q$ calculations. The authors are grateful to the referees for their constructive comments and suggestions.

\section{REFERENCES}

1. S. H. Talisa, M. A. Janocko, C. Moskowitz, J. Talvacchio, J. F. Billing, R. Brown, D. C. Buck, C. K. Jones, B. R. McAvoy, G. R. Wagner, and D. H. Watt, Low- and hightemperature superconducting microwave filters, IEFE. Trans. Microwave Theory Tech., 39:1448-1453 (1991)

2. F. A. Miranda, S. S. Tonich, and K. B. Bhasin, Performance of two-pole bandpass filters photodefined on double-sided Y-Ba-Cu-O and Tl-Ba-Ca-Cu-O thin films, Microwave Opt. Technol. Lett., 6:752-755 (1993)

3. G. C. Liang, R. S. Withers, B. F. Cole, S. M. Garrison, M. E. Johansson, W. Ruby, and W. G. Lyons, High-temperature superconducting delay lines and filters on sapphire and thinned $\mathrm{LaAlO}_{3}$ substrates, IHEF Trans. Appl. Supercond., 3:3037-3041(1993)

4. T. Konaka, M. Sato, H. Asano, and S. Kubo, Relative permittivity and dielectric loss tangent of substrate materials for high- $\mathrm{T}_{\mathfrak{c}}$ superconducting film, J. Supercond., 4:287, (1991) 
5. N. Newman and W. G. Lyons, High-temperature superconducting microwave devices fundamental issues in materials, physics, and engineering, .I. Suppercomd., 6:119-158 (1993)

6. J. Ceremuga, M. Barton, and F. Miranda, Analysis of influence of buffer layers on microwave propagation through high-temperature superconducting thin films, Supercond. Sicr. Technol. 7, (1994)

7. C. H. Mueller, F A Miranda, S. S. Tonich, and K. B Bhasin, YBCO X-band microstrip

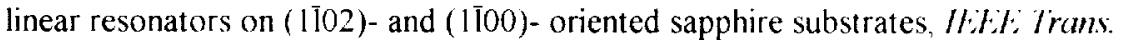
Appl. Sitpercond. 5:2559-2562(1995)

8. B. F. Cole, G C Liang. N Newman, K. Char, G. Zaharchuk, and J. S Martens, Largearea YBCO films on sapphire for microwave applications, Appl. Phys. Le't., 61:1727. $1729(1992)$

9. G. L. Matthaei, I. Young, and E. I T. Jones. "Microwave Filters, Impedance-Matching Networks, and Coupling Structures," Artech House Books, Dedham (1980) 